\title{
Erratum
}

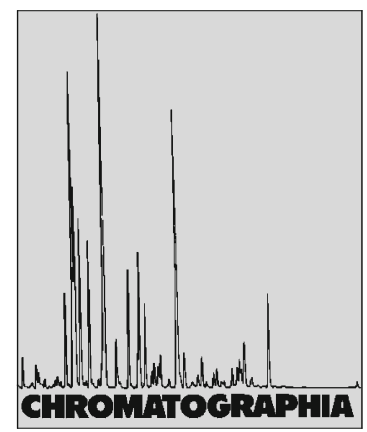

2008, 68, 155

\section{LC-MS-MS Study of the Pharmacokinetics of a 9- $\beta$-Dihydro-9, 10-O-acetal Derivative of Docetaxel in Rats and Beagle Dogs}

Xiaoling $\mathrm{Hu}^{1}$, Jianguo Sun ${ }^{1}$, Guangii Wang ${ }^{1, \bowtie}$, Xiaoyan Zhu', Gang Hao ${ }^{1}$, Yongchuan Gu², Frederik B. Pruijn ${ }^{2}$

${ }^{1}$ Key Laboratory of Drug Metabolism and Pharmacokinetics, China Pharmaceutical University, 24 Tong Jia Xiang Street, Box 210 Nanjing, Jiangsu 210009, People's Republic of China; E-Mail: ringse2004@163.com

2 Auckland Cancer Society Research Centre, Faculty of Medical and Health Sciences, The University of Auckland, Auckland, New Zealand

\section{Erratum to: Chromatographia (2008) \\ DOI 10.1365/s10337-008-0638-y}

Unfortunately, a wrong name is indicated as corresponding author

The correct corresponding author is

Guangii Wang

E-Mail: ringse2004@163.com 